\title{
ESPACIOS PÚBLICOS Y CONSTRUCCIÓN DE IDENTIDAD: LA EXPERIENCIA DE MONTES DE OCA
}

\section{PUBLIC SPACES AND IDENTITY CONSTRUCTION: THE EXPERIENCE OF MONTES DE OCA}

\section{Johnny Rodríguez-Gutiérrez ${ }^{1}$ \\ María Ruiz-Fuentes ${ }^{2}$ \\ Carlos Murillo-Zamora ${ }^{3}$}

Recibido: 16/07/2019

Aprobado: 11/11/2020

\section{RESUMEN}

El desarrollo y la planificación urbanística, en un mundo cada vez más globalizado y caracterizado por la comunicación digital y el predominio de las redes sociales, repercute directamente en la naturaleza de los espacios públicos -sobre todo cuando se piensa en términos del ágora griega- $y$ en la construcción de identidades en las comunidades; afectando, en forma directa, la participación ciudadana. En ese sentido, el espacio público en los condominios y en general en los barrios se constituye en un lugar en donde no necesariamente se produce la interacción entre personas para el debate público sobre los problemas comunitarios y la construcción de identidad. Pero, sobre todo, el espacio público es sustituido por aquel no público, que se sitúa en los centros comerciales y otros ámbitos no públicos. Frente a ello los Gobiernos locales no adoptan políticas públicas que fomenten y faciliten la interacción ciudadana. El cantón de Montes Oca, provincia de San José es un buen ejemplo de la forma en que se usan los espacios públicos hoy.

PALABRAS CLAVE: : ADMINISTRACIÓN PÚBLICA, POLÍTICA PÚBLICA, GOBIERNO MUNICIPAL, ESPACIO PÚBLICO, IDENTIDAD.

CLASIFICACIÓN JEL: H4, I3, ZO

1 Universidad de Costa Rica, Posgrado en Administración Pública; Código Postal: 11501-2060; San José, Costa Rica; johnnyrodriguezgutierrez@gmail.com

2 Universidad de Costa Rica, Posgrado en Administración Pública; Código Postal: 11501-2060; San José, Costa Rica; maria.rruizf@ gmail.com

3 Universidad de Costa Rica, Escuela de Administración Pública, Código Postal: 11501-2060; San José, Costa Rica; carlos. murillozamora@ucr.ac.cr 


\begin{abstract}
Development and urban planning, in a world increasingly globalized and characterized by digital communication and the predominance of social networks, has a direct impact on the nature of public spaces -especially when one thinks in terms of the Greek agora- and in the construction of identity in the communities; which affects citizen participation. In that sense, the public space in the condominiums, and in general in the neighborhoods, becomes a place where interaction between people does not necessarily take place for public debate about community problems. But, above all, the public space is replaced by the non-public space that is in the shopping centers and other non-public spheres. Faced with this, local governments do not adopt public policies that encourage citizen interaction. The Montes Oca canton is a good example of the way in which public spaces are used today.

KEYWORDS: PUBLIC ADMINISTRATION. PUBLIC POLICY. LOCAL GOVERNMENT. PUBLIC SPACE. IDENTITY

JEL CLASSIFICATION: H4, I3, Z0
\end{abstract}

\title{
I. INTRODUCCIÓN
}

La construcción de identidad, individual y colectiva, es clave en las relaciones sociales, políticas y económicas de toda comunidad. Se trata de un proceso en el que intervienen múltiples factores societales; pero su base es la diferenciación entre el yo/nosotros y el otro/otros; por lo tanto, se trata de cuestiones antropológicas, políticas, económicas, jurídicas y culturales; sin dejar de lado las políticas públicas que inciden en tal construcción.

Conforme ha aumentado el proceso de urbanización, generándose grandes ciudades $y$ metrópolis, $y$ en general conglomerados y colectivos humanos, en detrimento de lo rural e individual, surge lo relativo a los espacios públicos; pues existe una tendencia del sector inmobiliario a aprovechar al máximo la superficie (sobre todo en países como Costa Rica, en donde la tendencia histórica, por razones culturales, ha sido la preferencia de la construcción horizontal, y no la vertical, porque se aspira a que cada vivienda tenga su propio jardín y patio, aunque poco los edificios de apartamentos se construyen en el área Metropolitana (Prochile Costa Rica, 2018), descuidando el espacio público. Es decir, persiste la idea de que cada familia comparte en su casa de habitación y en su propio espacio; descuidando la relación de comunidad y la identidad barrial. Ello genera una contradicción en la construcción de la identidad comunitaria, que implica la vinculación al mismo tiempo que la diferenciación, lo cual influye en la participación ciudadana (Fundación Promotora de Vivienda, 2003, p. 4).

Por la división político-administrativa, en Costa Rica la gestión de esos espacios es responsabilidad de los Gobiernos locales, entendiendo que no se trata de la concepción decimonónica de la plaza o parque frente al templo, al palacio municipal y la escuela, que constituía el centro de referencia citadino, sino de un ámbito en el que se construyen identidades colectivas, se fomenta la ciudadanía y la participación en los asuntos públicos. En ese sentido, el espacio público constituye la esencia, identidad y carácter de la ciudad, el ágora griega; el centro de encuentro para el debate sobre temas comunitarios, para la actividades culturales y políticas, al igual que económicas, por los intercambios y planes que se pueden generar, como también deportivas, cuando se trataba de la plaza de futbol. Pero la expansión del área urbana -sin planificación con visión de comunidad y bajo la supervisión de las autoridades locales- alteró ese esquema, no solo porque la nueva dinámica económica trasladó ese centro a los espacios comerciales (los llamados "malls"); despersonalizando y hasta desapareciendo la construcción de lo comunitario y colectivo.

Tal situación condujo a un incremento del espacio privado. O para una mejor precisión al espacio no público, en referencia a las áreas comunes de los centros comerciales. Ello provocó una 
transformación de la ciudad, su forma y su estructura. Incluso los espacios públicos se redujeron, en el caso particular de los cantones de la Gran Área Metropolitana (GAM), a pequeñas áreas de juego (más conocidas como playground) dentro de las urbanizaciones y no necesariamente en la mejor ubicación del ámbito comunitario (Chávez, 2013).

A esto se sumó el problema de la creciente delincuencia, expresada particularmente en la forma de pequeñas pandillas o grupos de delincuentes comunes, y de la presencia de grupos del crimen organizado, sobre todo dedicados al narcotráfico al menudeo (Zúñiga, Corrales \& Vargas, 2019). Así se incrementó la inseguridad ciudadana y la ciudadanía se refugió en sus casas enrejadas o en los centros comerciales, desapareciendo -en la práctica- el espacio público como lugar de encuentro y construcción de identidad, dando lugar a una que se denomina una "ciudad fragmentada", que en Costa Rica comenzó a observarse hacia finales del siglo XX como parte del fenómeno de la "segregación socioespacial" (Fumero, 2009, p. 9). Esa situación hace que Mazza (2009, p. 4) señale que la ciudad “...representa, el medio natural donde el miedo por la criminalidad se difunde y donde se despliegan sus efectos; bajo este desafío, la ciudad está cambiando profundamente en las formas, en las modalidades organizativas, en los comportamientos individuales y colectivos".

Cuando la construcción de identidad, individual o colectiva, está condicionada por la díada seguridad/inseguridad, el proceso de torna más complejo, pues la seguridad constituye un bien público puro, que debe ser garantizado por la autoridad para el disfrute de los individuos y colectividades. Y como se trata de una cuestión de percepción, la gente disfruta de ella, sobre todo, en el espacio público. Así la seguridad tiene que ver con condiciones históricas, políticas, sociales y culturales (Mazza, 2009, p. 4), que se manifiesta en los espacios colectivos.

Por eso, hoy, no se trata solo de urbanización y espacios públicos de la ciudad como el centro de referencia para la construcción de identidad. Es necesario considerar la dimensión urbana: tamaño, densidad y heterogeneidad (Mazza, 2009, p. 6). Pues como sucede en la GAM las ciudades forman parte de una gran masa urbanística, integrada más allá de los límites cantonales; así algunas ciudades pueden estar más vinculadas con otra del cantón vecino, que con la cabecera de la división administrativa a la que pertenece.

Cada vez más se habla de la crisis de la ciudad y de lo urbano, por la desigualdades económicas, sociales y espaciales (Gallegos, 2011), dando lugar a una tendencia inversa: la búsqueda del espacio rural para escapar a los peligros de la urbe. Pero sin que esto signifique regresar al espacio de producción agrícola, sino aquel que está fuera de la urbe y un extenso paisaje, de la gran masa urbanística, con poco espacio individual/familiar; eso sí, con los servicios públicos y privados al alcance de la mano, bajo la tesis de "ciudades rurales" y la "nueva ruralidad" y encadenamientos urbano-rurales (Rosas-Baños, 2013). Sin embargo, la dependencia socioeconómica de los centros de trabajo, producto del crecimiento demográfico urbano y la reclasificación de los espacios rurales impedirá que se revierta la tasa de urbanización (Cunha, 2002, p. 7). Ello se manifiesta en una tendencia a la reducción del área disponible para urbanizar, lo cual genera la búsqueda de las empresas constructoras para aprovechar al máximo el área de construcción destinada a vivienda, en detrimento del espacio compartido y, por ende, una mayor dificultad para generar el espacio social y garantizar el debate de la ciudadanía sobre los problemas comunes, según un estudio de la Fundación Promotora de Vivienda (FUBROVI, 2018), El Plan Nacional de Ordenamiento Territorial establecen como punto de equilibrio $10 \mathrm{~m}^{2}$ de espacio público por persona, lo cual no siempre se cumple (FUBROVI, 2018, p. 74). Sin duda, ello tiene repercusiones en la participación ciudadana y en el ejercicio de la democracia.

El cantón de Montes de Oca constituye un buen ejemplo de los cambios que enfrentan los espacios públicos, por tratarse de un municipio que en 2011 tenía el $100 \%$ de población urbana, en contraste con el 95.7\% del año 2000 (Programa Estado de la Nación en Desarrollo Humano Sostenible, 2013, p. 42), en el cual el 86,5\% de la población se dedica al sector terciario; además, 
cuenta con un número importante de centros de educación (primarios, secundarios y terciarios). Por ello lo utilizamos como ejemplo para abordar la problemática del espacio público y la construcción de identidad.

En este artículo se analizan las acciones en materia de recuperación de espacios públicos realizadas por el Gobierno local del cantón de Montes de Oca y se estudia, la relación entre los espacios públicos y la identidad cantonal.

En la primera sección se abordan algunas aproximaciones conceptuales que son necesarias para comprender el tema objeto de análisis. En una segunda sección se resumen algunas de características particulares del cantón de Montes de Oca y sus espacios públicos. Así en la tercera sección hacemos referencia a la formulación e implementación de políticas públicas en esta materia.

\section{Algunas aproximaciones conceptuales}

Las ciudades, sean urbanas o rurales, son un elemento fundamental para facilitar y garantizar el cumplimiento de los derechos humanos, tanto sociales, políticos, económicos y culturales, porque estos tienen un gran impacto en el nivel local (según los planteamiento de la Comisión de Inclusión Social, Democracia Participativa y Derechos Humanos de la organización española Ciudades y Gobiernos Locales Unidos -CGLU-), pues se considera que al adoptar un enfoque basado en derechos, los Gobiernos locales contribuyen a la calidad de vida y la ciudadanía plena ${ }^{4}$. En ese contexto el Consejo Mundial de CGLU adoptó en 2011 la Carta-Agenda Mundial de Derechos Humanos en la Ciudad, en la que se establece "la ciudad como un derecho". Entonces, hay una estrecha relación entre derechos, ciudad y la de construcción de identidad y ciudadanía. En este sentido, la ciudad "se constituye en la agregación de espacios públicos y privados que generan una identidad común para la población que la habita” (Dammert, 2004, p. 6). Mientras que la ciudadanía es "...un status [sic], es decir, un reconocimiento social y jurídico por el cual una persona tiene derechos $y$ deberes por su pertenencia a una comunidad, en general, de base territorial y cultural" (Borja, 2001).

Y de manera breve, pero consideramos necesaria una referencia en el marco de la relación ciudad, espacios públicos y derechos humanos, está la cuestión del reconocimiento de los derechos humanos a los migrantes modernos -lo cual es visible en Montes de Oca, por la cantidad de personas que residen temporalmente- y genera el fenómeno cosmopolita de la "ciudadanía universal" y el respecto a los derechos humanos universales a todos los individuos más allá de su lugar de residencia (Estévez, 2016). Y la ciudadanía es, como se indicó arriba, una "adscripción a una comunidad política determinada" tiene que ver con construcción de identidad y con diferencias culturales y sociales, al mismo tiempo que con exclusión/inclusión (Estévez, 2016, pp. 62-62).

Desde la perspectiva anterior, el espacio público es fundamental en la concepción de la ciudad, al ser considerado el lugar de intercambio, interacción, convivencia y construcción de tejido social y, por ende, de identidad comunitaria. Al respecto, Borja y Muxí (2003) manifiestan que la historia de la ciudad es la de sus espacios públicos y afirma que estos promueven la cultura urbana y la ciudadanía. Esto no es novedoso, porque como lo mencionamos arriba, el ágora griega (del griego à $\gamma o \rho a ́$, asamblea, de ả $\gamma \varepsilon i \rho \omega$, 'reunir') cumplía esa función, que luego desapareció y poco a poco cedió lugar al espacio no público y con mayor fuerza al espacio privado.

Además, se concibe el espacio público como un área facilitadora de buenas relaciones que permiten resolver diversas problemáticas de manera colectiva (Borja \& Muxí, 2003; Segovia, 2007). También es preciso señalar que estos espacios pueden convertirse en el fiel reflejo de las crisis económicas, sociales y culturales que afrontan las ciudades, traduciéndose así en la manifestación

4. . Consúltese las ideas sobre Gobiernos locales y promoción y protección de los derechos humanos, "Ciudades por los Derechos" en https:/www.uclg-cisdp.org/es/actividades/ciudades-de-derechos-humanos 
de las problemáticas del territorio en un momento histórico determinado. Como el escenario de la dialéctica y lo dialógico, que respalda la acción institucional y la iniciativa ciudadana (Pindado \& Rebollo, 2015). Por eso, Pindado y Rebollo (2015, p. 67) consideran que el debate público que permite que los y las vecinas de un municipio promuevan actividades culturales a través de la puesta en marcha de "...un proceso comunitario que bien puede acabar dando lugar a una organización o asociación comunitaria”, nacida en el espacio público y no el privado, que tiene una naturaleza restrictiva.

Con respecto a la incidencia del espacio público en la construcción de identidad, debe tomarse en cuenta que, según Hiernaux (citado por Sánchez \& Domínguez, 2014, p. 41) en los procesos de construcción de la identidad, el espacio socio-geográfico juega un papel decisivo, debido a que tradicionalmente los grupos sociales construyen su identidad a partir de un espacio donde inscriben sus actividades y que modelan por medio de signos que permiten identificar $y$ diferenciarlos de los de otros. Esto ocurre tanto en lo relativo a la identidad individual, como colectiva y comunitaria.

Sobre este tema, se debe valorar que las dinámicas sociales, se han transformado abruptamente en los últimos años, en donde el auge de las tecnologías móviles y el modelo de desarrollo económico han imposibilitado potenciar el aprovechamiento del espacio público, la comprensión de lo privado y la "identidad digital" (Di Siena, 2010), por lo que se cuestiona si son necesarios en un mundo de redes sociales (Magalhães, 2014). Sobre ello Magalhães (2014) señala que más allá “...de la enorme oferta de virtualidad en nuestras vidas —Facebook, Skype, Twittersiguen siendo las ciudades y sus espacios cívicos — escuelas, centros comunitarios, oficinas, tiendas, espacios públicos - los lugares por excelencia que permiten el encuentro real con los otros", porque las ciudades generan "círculos virtuosos" que mejoran la vida comunitaria y, agrega, "el éxito de una ciudad depende en gran medida de su capacidad de promover la cohesión social".

En el caso particular de Montes de Oca, al consultarse con las autoridades locales durante las entrevistas, no se encontró una política pública que articule las intervenciones en materia de espacios públicos y que permita tomar en cuenta estos aspectos y generar transformaciones reales en el uso y aprovechamiento del espacio público y en la convivencia de la población. Así se afecta la cohesión social necesaria para la construcción de identidad y la participación ciudadana.

Tomando en cuenta lo anterior, es que se determina que la identidad está asociada con las relaciones de las personas y su entorno (físico y social) y que por tanto el espacio, debe pasar por un proceso de humanización que en gran medida definirá su éxito o fracaso a la luz de las necesidades de quienes lo habitan. Adicionalmente, es necesario considerar que la internalización de las relaciones sociales y espaciales, así como la satisfacción de necesidades, que intervienen en la construcción identitaria propician la adopción de símbolos sociales, los cuales adquieren un significado y una aceptación colectiva (Ávila, 2017, pp. 19-120).

Sobre la construcción de identidad Vicherat (2007, p. 59) afirma que esta "es un proceso que se define $y$ adquiere forma de manera relacional y es producto de relaciones sociales, que se generan a través de la experiencia que cada sujeto tiene al estar con otros", por lo que, agrega "...es a través de las experiencias cotidianas de estar entre otros que cada uno de nosotros hace presente su propia identidad".

Además, debe considerarse que los usos y costumbres que acontecen en los espacios públicos son un parámetro para determinar los grados de cohesión e integración social, uso y apropiación, convivencia, sentido de pertenencia de las personas y la capacidad que tienen los espacios públicos para generar ciudadanía e identidad cantonal.

En la construcción de esa ciudadanía e identidad cantonal intervienen diversos factores, entre los cuales se pueden citar: la participación social, la apropiación del espacio, el apoyo comunitario, el nivel de percepción de inseguridad y las acciones sociales realizadas en los espacios públicos. También inciden otros elementos tales como: limpieza, accesibilidad, estética, comodidad 
y la seguridad de los espacios públicos. Estos son criterios que se toman en cuenta para determinar si los espacios públicos fortalecen el sentido de comunidad y la cohesión social. Sin embargo, la relación entre espacio público y comunidad no se produce se forma espontánea, y por el contrario se debe recurrir a políticas públicas que favorezcan el desarrollo de un sentimiento de identidad comunitaria (Vargas \& Merino, 2012, 902).

Por otra parte, Segovia (2005) señala que la existencia de aspiraciones colectivas, junto a los niveles de confianza y asociatividad, constituyen ingredientes del capital social que determina la calidad de la vida de las personas y de las comunidades. Reiteramos, que la espontaneidad no resulta hoy el atributo dominante en la generación de la comunidad como ámbito de interacción colectiva.

Los conceptos de espacio público e identidad cantonal son concebidos como construcciones sociales e históricas que se nutren de las tradiciones, vivencias y de la cotidianidad de los diferentes grupos etarios que conforman el cantón; pero que requieren el impulso de estrategias por parte de las autoridades locales (Ávila, 2017). Con el tiempo, esos constructos se van modificando según las expectativas de los ciudadanos, la visión del gobierno local, de la interacción entre lo público, lo privado y las comunidades, así como de las diferentes disciplinas además de la administración pública, tales como arquitectura, sociología, planificación urbana, que intentan definirlos desde distintos enfoques.

Desde la Administración Pública, el espacio público es un activo del Estado que puede potenciar el desarrollo de actividades sociales, culturales y la economía local. Además, se identifica como un lugar donde se puede construir tejido social a partir de la diversidad, la convivencia, las afinidades y las diferencias. Pero, como los hemos reiterado, tal construcción requiere de políticas públicas dirigidas y articuladas a la consecución de resultados concretos. Desde esta perspectiva, Duhau y Giglia (2010) señalan que los abusos de los particulares sobre los espacios públicos obedecen a una gestión gubernamental deficiente, incierta y contradictoria de las normas, con controles esporádicos y débiles por parte de las administraciones locales. Es decir, la construcción de identidad, el debate público y la generación de acciones colectivas no resulta de la simple interacción de un grupo de individuos que se encuentran en un lugar de reunión; se requiere una intencionalidad, $y$ esta debe ser impulsada por las autoridades locales.

Es en esta coyuntura, en la cual las políticas públicas se consolidan como un instrumento que puede orientar la gestión del Gobierno local para resolver las problemáticas sociales que limitan la construcción de identidad cantonal, que se puede aprovechar los espacios públicos como potenciadores de mayores niveles de bienestar social, económico y cultural. Al mismo tiempo que fomentar la participación ciudadana en la gestión de lo público, contribuyendo al fortalecimiento de la democracia.

\section{METODOLOGÍA}

Para identificar las acciones de recuperación de espacios públicos desarrolladas por la Municipalidad de Montes de Oca, fue necesario visitar las comunidades para conocer las condiciones y problemáticas de estos espacios, el uso y aprovechamiento por parte de la comunidad y así determinar si existía interacción entre la sociedad, el gobierno local y otras organizaciones públicas y privadas.

Se utilizaron técnicas para investigación cualitativa como la observación y talleres con las comunidades seleccionadas para identificar las necesidades de la población con respecto al uso de los espacios públicos, así como entrevistas semi estructuradas a contactos de interés y revisión documental. Se elaboró una matriz de congruencia para identificar información relevante para el desarrollo de los objetivos.

En el trabajo de campo se entrevistó a funcionarios de la Municipalidad de Montes de Oca $y$ otras personas vinculadas con iniciativas de espacios públicos en el cantón. Entre las personas 
entrevistadas estuvieron el arquitecto Gillio Francesa, director de la Oficina de Planificación Urbana; Gerardo Madrigal Aguilar, coordinador de la Oficina de Desarrollo Social; $y$ otras personas funcionarias que forman parte de la Comisión de Espacios Públicos de la Municipalidad. Además, se obtuvo información por parte de Marcela Vargas, líder comunal del distrito de San Rafael; Edgar Chacón, presidente de la Asociación de Desarrollo de la Urbanización Málaga en Sabanilla de Montes de Oca; y Julio Téllez, presidente del Comité Cantonal de Deportes y Recreación de Montes de Oca.

Se realizaron dos talleres denominados "El espacio público y su relación en la construcción de una identidad cantonal", a los cuales se invitó a dirigentes comunales de los cuatro distritos, con el propósito de conocer la percepción de estos con respecto a los espacios públicos, las condiciones actuales, las necesidades y expectativas distritales. En estos talleres participaron 13 personas. Es necesario se señalar que no se obtuvo respuesta por parte de los distritos de Mercedes y San Pedro.

Los talleres se estructuraron de la siguiente manera: una actividad de presentación, denominada la telaraña; una presentación introductoria a la investigación; y posteriormente, se dividió el grupo en equipos de trabajo con el fin de que estos a partir de una serie de preguntas generadoras identificaran, las acciones en materia de recuperación de espacios públicos implementadas por la Municipalidad de Montes de Oca, manifestaran las necesidades locales y generaran propuestas de abordaje a las mismas. Una vez concluida la dinámica en los diferentes grupos se llevó a cabo una plenaria para conocer el trabajo realizado.

En la segunda parte del taller, se utilizó la técnica de la lluvia de ideas y un ejercicio de reflexión sobre la participación ciudadana en la construcción del espacio público. Lo anterior, con el objetivo de delimitar el concepto de espacio público y su relación en la construcción de una identidad cantonal. Sobre este tema, fue de interés conocer si las acciones municipales que se han dado hasta el momento han atendido las necesidades de las personas habitantes del cantón, en materia de seguridad ciudadana, recreación y condiciones de acceso. Esta actividad permitió, también, identificar las oportunidades de mejora de los espacios públicos, a partir de los elementos que los participantes del taller consideran que forman parte de la identidad del cantón.

Finalmente, la última fase de los talleres consistió en la aplicación de un METAPLAN para brindar recomendaciones asociadas con la implementación de políticas públicas para la recuperación de espacios públicos, a partir del análisis de la experiencia de la Municipalidad de Montes de Oca. Como parte de esta actividad se buscó determinar las acciones prioritarias para mejorar las condiciones de los espacios públicos, los criterios a considerar por parte del gobierno local para realizar una intervención en estos y el rol que desempeñan las comunidades en las intervenciones de los espacios públicos.

Los temas desarrollados en los talleres se vinculan de manera directa con la definición del problema y los objetivos de la investigación, la cual buscaba delimitar teóricamente lo que comprende el concepto de espacio público y su relación en la construcción de una identidad cantonal. Además, se pretendía identificar las acciones en materia de recuperación de espacios públicos implementadas por la Municipalidad del cantón de Montes de Oca para determinar los efectos que esas acciones habían generado en la construcción de una identidad cantonal.

Además, se seleccionó una muestra de espacios públicos a partir del inventario suministrado por el Departamento de Catastro de la Municipalidad de Montes de Oca. Con base en esa información y los objetivos del trabajo, se seleccionaron los parques, plazas y áreas comunales porque son lugares donde pueden concurrir las personas habitantes del cantón para desarrollar actividades sociales, familiares, recreativas e integración que permiten construir identidad cantonal y mayores niveles de participación ciudadana, generando así sentido de pertenencia, respeto a la diversidad e incidencia en la solución a problemáticas sociales. La muestra se seleccionó considerando la cantidad de población de cada distrito y es proporcional a la cantidad de espacios púbicos que posee.

Una vez seleccionados los espacios públicos, se realizó un mapeo de las organizaciones sociales presentes en cada distrito, ubicando así las Asociaciones de Desarrollo Cantonales, los 
Comités de vecinos, la Unión Cantonal de Montes de Oca y el Comité Cantonal de Deportes de Montes de Oca. Para cada distrito se seleccionaron aquellas organizaciones que representan a las comunidades con mayor cantidad de espacios públicos y la población por distrito, según datos del gobierno local.

Por otra parte, se consultaron 69 fuentes de información documental para dar sustento teórico al proceso y desarrollar los objetivos de la investigación. Lo anterior, permitió disponer de una base teórica y práctica para generar recomendaciones asociadas con la implementación de políticas públicas para la recuperación de espacios públicos a partir del análisis de la experiencia de la Municipalidad del cantón de Montes de Oca.

A partir de esta información y con la identificación de los elementos asociados con la identidad cantonal, se logró brindar una propuesta para la formulación de una política pública, que incorporara esta perspectiva en las acciones en materia de recuperación de espacios públicos.

Como limitantes de la investigación se debe mencionar que no hubo participación ciudadana de ninguna de las organizaciones comunales seleccionadas en la muestra de los distritos de San Pedro y Mercedes debido a las malas experiencias en el pasado con estudios similares que ha realizado la Municipalidad de Montes de Oca.

\section{RESULTADOS}

\section{Una breve caracterización de Montes de Oca y el espacio público}

Para el caso particular de Montes de Oca, se identifica que la población no considera que exista un parque central, como espacio público culturalmente reconocido como punto de encuentro para la población. Este hallazgo presenta una gran limitante para la consolidación de una identidad cantonal. Además, constituye una ruptura con la estructura urbanística que se puede considerar tradicional de lo que fueron las aldeas en el siglo XIX y que poco a poco evolucionaron hacia las villas y luego a las ciudades.

Adicional a lo anterior, los habitantes del cantón consideran que las identidades propias de cada distrito se han ido debilitando como resultado de la falta de arraigo y de la nula o escasa convivencia entre distintas generaciones. Otro aspecto para tomar en cuenta son las características particulares de la población del cantón, en donde la migración a y desde otras zonas del país diluye la posibilidad de conservar o potenciar una identidad cantonal, y sobre todo identidad distrital. De ahí que cabe cuestionarse si no existe una tendencia a la pérdida de tal colectividad, dando paso a otro tipo de identidades, en perjuicio de la ciudadanía y la comunidad.

Por otro lado, no es posible negar la relevancia que adquiere la presencia de la Universidad de Costa Rica (UCR) para Montes de Oca. Sobre este tema no solo se reconoce el aporte de esta institución en la formación de profesionales que inciden en el desarrollo económico, social y cultural de las comunidades, sino también de las transformaciones que genera mediante la investigación y la acción social. Esta situación ha llegado a que se identifique más la Ciudad Universitaria Rodrigo Facio y la UCR como el referente identitario, en claro detrimento de lo cantonal. Es decir, San Pedro como distrito 1 del cantón es conocido por la universidad y no por la cabecera cantonal; esto conduce a que los otros distritos: Sabanilla, Mercedes y San Rafael, adquieran cierto grado de autonomía como comunidad.

Ahora bien, con respecto a las acciones realizadas por la Municipalidad de Montes de Oca para la recuperación de espacios públicos, se concluye que estas no han contribuido a la construcción de identidad cantonal. Lo anterior, debido a que como resultado del proceso de consulta comunal en el cual se determinó que hay una escasa convivencia entre la población -está ausente la cohesión social y la construcción identitaria- $y$ un nulo sentido de pertenencia y apropiación de los espacios públicos. Además, la población considera que falta voluntad política por parte de las autoridades del Gobierno 
local para resolver problemáticas alrededor de los espacios públicos, lo cual se agrava con la poca participación ciudadana y las deficiencias en el diseño de los proyectos que se desarrollan en algunas ocasiones sin un proceso de consulta a las comunidades. Así existe un "círculo vicioso" que favorece el uso de los espacios no públicos y privados, dificultando el desarrollo del sentido de comunidad.

Por otro lado, las principales necesidades identificadas por las comunidades alrededor de los espacios públicos están asociadas con condiciones de diseño, tales como una adecuada iluminación que permita mejorar la seguridad, la instalación de mobiliario para realizar actividad física, comodidades para las personas adultas mayores, la organización de actividades recreativas que permitan unir a las familias y mayor vigilancia por parte de la fuerza pública. Las apreciaciones anteriores fueron externadas por las personas entrevistadas, las cuales reconocen a los espacios públicos como lugares de encuentro informal y no, como un auténtico escenario de debate comunal.

Las condiciones de los espacios públicos y la cantidad de intervenciones ${ }^{5}$ realizadas en los diferentes distritos del cantón son características que inciden en la realidad de los distintos barrios y caseríos y que incluso se reflejan en el índice de desarrollo social del cantón. Por ejemplo, San Rafael de Montes de Oca es el distrito con la menor cantidad de intervenciones y de espacios públicos, $y$ los habitantes consideran que esto se debe principalmente a que la participación ciudadana es muy limitada, lo cual imposibilita el planteamiento de iniciativas innovadoras ante los Concejos de Distrito.

En ese sentido, en términos generales los lugareños perciben que San Rafael es el distrito abandonado y se le reconoce como una zona de paso. Además, los habitantes del distrito manifiestan que el desarrollo de esta comunidad giró alrededor de los programas de desarrollo social que realizó el Estado en el ámbito nacional en los años 1970. Para el año 2017, según datos del Ministerio de Planificación Nacional y Política Económica (MIDEPLAN, 2017, pp. 10-12) el distrito de San Rafael presentaba el menor Índice de Desarrollo Social $(77,43)$, el distrito con el mayor índice fue Mercedes con un 90,85, seguido de Sabanilla con 89,24 y en tercer lugar se ubica San Pedro con un 88,39.

En términos generales la mayoría de las personas consultadas coincidieron en que para promover mayor apropiación y lograr de manera paulatina la reconstrucción del tejido social, es fundamental garantizar adecuadas condiciones de acceso y asegurar las medidas mínimas para el uso y disfrute de los espacios públicos. De esta manera se propicia mayor confianza e involucramiento en actividades para propiciar el desarrollo social, económico, cultural y político de las comunidades.

\section{La situación de Montes de Oca en términos del espacio público}

El Gobierno local, según la información recolectada a través de las entrevistas a la ciudadanía y a funcionarios municipales, no tiene visión social de los espacios públicos que permita visualizarlos como pilares para la construcción de la identidad cantonal y el tejido social, no hay un norte que oriente el accionar del gobierno local en esta materia ni tampoco se realizan intervenciones de manera articulada, lo cual se evidencia principalmente al no existir una política pública en esta materia. Al respecto, una política pública sobre el espacio público se constituiría en un instrumento para identificar las necesidades, definir la inversión de los recursos y potenciar el desarrollo integral del cantón.

Sobre este tema, las personas funcionarias municipales entrevistadas señalan que esta situación surge a raíz del manejo político y de la diversidad ideológica presente en la conformación

5 Las intervenciones de los espacios públicos son concebidas tanto como acciones de mejora a la infraestructura de la ciudad, lo cual podría no generar mayor impacto, hasta la ejecución de estrategias que buscan complejizar la problemática que existe en el territorio y lograr transformaciones sociales mediante la participación ciudadana y la implementación de políticas públicas que atiendan las necesidades manifiestas de la población. 
del Concejo Municipal, que en ocasiones puede ser un limitante para el desarrollo de algunos proyectos o iniciativas de las comunidades, debido a que se anteponen las posturas políticas a los intereses de la población.

Como parte de este proceso, se determina que el Comité Cantonal de Deportes (CCD) de Montes de Oca es un actor clave en la recuperación de los espacios públicos, y que únicamente se requiere una mayor coordinación entre este órgano y el Gobierno local para que las intervenciones que realiza no se den de manera aislada. En el caso específico del CCD, el presidente de este señala que se ha intentado contribuir en la construcción de la identidad cantonal por medio del fomento de actividades deportivas y recreativas; no obstante, la principal limitante ha sido la lenta respuesta del Gobierno local para resolver algunas problemáticas o coordinar la ejecución de acciones.

Adicionalmente, la mayoría de las acciones realizadas por el Gobierno local y por el CCD se han orientado a mejorar la estética y la instalación de mobiliario para realizar actividad física, sin que esto garantice la construcción de la identidad, porque no se ha logrado la integración de la comunidad que permita el debate público y la participación ciudadana. Si bien cada grupo etario podría plantear distintas demandas, tanto la población más joven como la adulta mayor, coincidieron en la necesidad de contar con mobiliario para realizar actividad física.

En relación con las intervenciones realizadas en materia de recuperación de espacios públicos y a partir de las entrevistas realizadas, el equipo investigador identificó que no existe articulación entre las distintas instancias del Gobierno local y un órgano adscrito al mismo, como lo es el CCD, por lo que es aún menos probable que se establezcan alianzas con otras organizaciones públicas o privadas. Sobre este tema, tanto a nivel interno de la municipalidad, como desde la perspectiva de algunas de las personas entrevistadas, no existe una acción o comunicación estratégica dentro de la instancia cantonal, lo cual se refleja en las relaciones que se establecen entre la Dirección de Planificación Urbana y la Oficina de Desarrollo Social; al ser estas dos instancias esenciales para definir las propuestas de intervención y potenciar la satisfacción de las necesidades de la población de los diferentes distritos.

A pesar de lo anterior, las comunidades perciben que la Alcaldía está haciendo todos los esfuerzos necesarios para que se mejoren las condiciones de los espacios públicos; no obstante, se afirma que el problema también se encuentra en los mandos medios que entraban las iniciativas de las comunidades. Al respecto, según la opinión de funcionarios municipales, con quienes se conversó, $y$ de las entrevistas y talleres realizados a dirigentes comunales, se concluye que existen debilidades en la gestión municipal, y que hay poco compromiso y doble discurso por parte de las personas funcionarias para resolver situaciones alrededor de los espacios públicos.

Por otra parte, el Departamento de Obras de la Municipalidad de Montes de Oca no posee la capacidad requerida para dar acompañamiento constante a las comunidades en la formulación de iniciativas y proyectos que se presente ante los Concejos de Distrito. En este sentido, no existe una oficina que administre un portafolio que disponga de prioridades de intervención previamente analizadas que puedan iniciarse cuando la administración disponga de algún remanente presupuestario.

Sobre el tema planteado anteriormente, algunas de las personas entrevistadas afirmaron que las experiencias en la recuperación de espacios públicos han sido "quijotescas" por la falta de apoyo del Concejo Municipal. Las comunidades perciben que se reproduce un discurso vacío sobre la importancia que tienen estas áreas en el mejoramiento de las condiciones sociales, económicas, políticas y culturales del distrito y en general del cantón. Asimismo, advierte la ciudadanía que tampoco se desarrollan, por parte de las autoridades, iniciativas para formular estrategias de intervención.

En virtud de la ausencia de una política pública, las personas funcionarias del Catastro Municipal, consideran necesario valorar el rol que desempeñan las empresas inmobiliarias en el cantón, quienes deben disponer por cada una de las obras de un 10\% del territorio para espacios 
públicos; no obstante, en ocasiones las áreas destinadas son poco aprovechables, esto, a pesar de que cumplen con las disposiciones definidas en la Ley de Planificación Urbana y en el Reglamento de Control de Fraccionamiento y Urbanizaciones. $O$ en el mejor de los casos se ubican dentro de los desarrollos urbanísticos, especialmente condominios con acceso único y regulado, lo que los convierte en espacios públicos solo para el disfrute de quienes habitan el proyecto y no para todos los habitantes del distrito.

Algunos funcionarios municipales y dirigentes comunales perciben que el modelo de gestión del Gobierno local no permite visualizar a los espacios públicos como creadores de identidad cantonal, por la falta de innovación en la gestión. En este sentido, se percibe que el Gobierno local se concentra en actividades ordinarias como la recolección de desechos y residuos, el trámite de permisos de construcción y patentes, desarrollo de infraestructura vial de rutina y el mantenimiento de cementerios.

De lo anterior es posible afirmar que no hay desarrollo organizacional que permita alinear los mandatos legales que tiene la organización con las crecientes demandas ciudadanas alrededor de los espacios públicos. Esto ha dificultado que la municipalidad pueda visualizarlos como potenciales generadores de desarrollo social, económico, cultural y político, trabajando de manera coordinada con diversos actores y considerando los recursos disponibles. Por consiguiente, la problemática relacionada con la existencia o no, y lo apropiado o no, de los espacios públicos, permite señalar que su mera existencia no es garantía de que se constituya en el ágora griega y que sirva de elemento para construir identidad y fomentar la participación ciudadana. Para establecer tal relación son necesarias acciones por parte de las autoridades locales.

\section{Sobre una política pública para la recuperación de los espacios públicos}

Con base en la información obtenida de las personas y servidores públicos entrevistados, quienes desarrollamos esta investigación consideramos que es necesaria la articulación de acciones y/o procesos a lo interno de la Municipalidad de Montes de Oca. En este sentido, una política cantonal de espacios públicos debe tener como norte lo establecido en el artículo 50 de la Constitución Política, según el cual: "Toda persona tiene derecho a un ambiente sano y ecológicamente equilibrado". Por ello, está legitimada para denunciar los actos que infrinjan ese derecho y para reclamar la reparación del daño causado. El Estado garantizará, defenderá y preservará ese derecho".

La política pública que se formule en el cantón debe garantizar el cumplimiento del principio de participación ciudadana para resolver de manera colectiva los problemas asociados a los espacios públicos, así como la posibilidad de propiciar el desarrollo social, económico y cultural del cantón y asegurar intervenciones que reúnan los valores, costumbres, usos y preferencias del cantón. Además, debe contribuir al cumplimiento de los Objetivos del Desarrollo Sostenible, específicamente el número 11 sobre Ciudades y comunidades sostenibles, que define a las ciudades como hervideros de ideas, comercio, cultura, ciencia, productividad $y$ desarrollo social ${ }^{6}$.

6. De acuerdo con el Programa de las Naciones Unidas para el Desarrollo (2018) el objetivo 11 sobre Ciudades y comunidades sostenibles tiene en cuenta que:

Más de la mitad de la población mundial vive hoy en zonas urbanas. En 2050, esa cifra habrá aumentado a 6.500 millones de personas, dos tercios de la humanidad. No es posible lograr un desarrollo sostenible sin transformar radicalmente la forma en que construimos y administramos los espacios urbanos.

El rápido crecimiento de las urbes en el mundo en desarrollo -como resultado de la creciente población y del incremento en la migración- ha provocado un incremento explosivo de las mega urbes, especialmente en el mundo desarrollado, y los barrios marginales se están convirtiendo en una característica más significativa de la vida urbana.

Mejorar la seguridad y la sostenibilidad de las ciudades implica garantizar el acceso a viviendas seguras y asequibles y el mejoramiento de los asentamientos marginales. También incluye realizar inversiones en transporte público, crear áreas públicas verdes y mejorar la planificación y gestión urbana de manera que sea participativa e inclusiva (disponible en https:// www.undp.org/content/undp/es/home/sustainable-development-goals/goal-11-sustainable-cities-and-communities.html). 
Las acciones en materia de recuperación de espacios públicos deben responder a una política formulada a partir de un proceso de participación ciudadana donde se discutan las necesidades de cada distrito $y$ que se orienten a generar arraigo que permita mayor sentido de pertenencia $y$ un mayor uso y aprovechamiento de manera sostenible.

La formulación e implementación de la política también debe considerar la capacidad instalada del sistema de gestión institucional del Gobierno local, el equilibrio de fuerzas entre actores internos (autoridades, direcciones, departamentos y oficinas) y externos (comunidades, organizaciones sociales y privadas), los resultados de las experiencias previas en la formulación de una política cantonal de recuperación de espacios públicos, la capacidad financiera y la posibilidad de realizar alianzas con otros sectores que se puedan involucrar en el proceso.

Asimismo, este instrumento de planificación debe contemplar la posibilidad de que, si un espacio no tiene las condiciones para ser aprovechable, el Gobierno local pueda realizar inversiones mínimas que permita desarrollar posteriormente intervenciones más complejas con los recursos disponibles. En este sentido, se debe invertir a partir de un enfoque de derechos que garantice el uso libre, democrático y equitativo por parte de las personas usuarias; pero con el propósito de fomentar la construcción de identidad comunal y la participación ciudadana.

Por lo anterior, se requiere involucrar a las comunidades en el diseño y conservación de los espacios públicos para generar arraigo, apropiación, sentido de pertenencia y responsabilidad por el buen uso de las instalaciones. Para diseñar las intervenciones en los espacios públicos, no solo es necesaria la participación ciudadana, se requiere también del conocimiento de aspectos técnicos que deben cumplir las propuestas canalizadas a través de los Consejos de Distrito y aprobadas por el Concejo Municipal y la Alcaldía. En este sentido, se recomienda la creación de guías para diseño de espacios públicos, que sirvan como material de consulta para las comunidades cuando plantean iniciativas de intervención. Estas guías les permitirán conocer los requerimientos solicitados por la Municipalidad de Montes de Oca y lograr una mayor articulación de procesos entre el Departamento de Planificación Urbana y la Oficina de Desarrollo Social, con el objetivo de verificar que el diseño de los proyectos responda a las problemáticas insatisfechas en las comunidades.

De acuerdo con las personas funcionarias entrevistadas por parte de la Municipalidad, Montes de Oca no cuenta con la capacidad instalada necesaria para dar mantenimiento preventivo y correctivo a los espacios públicos y con ello garantizar condiciones adecuadas para el uso de estas áreas, se considera necesario establecer alianzas estratégicas con organizaciones comunales para asumir esta responsabilidad de manera compartida, tal y como lo gestionaron algunas comunidades del cantón, a saber: Málaga, Cedros, entre otras, para el desarrollo de tareas o actividades concretas.

La política no puede desconocer el contexto económico, social, cultural y político del cantón, ni tampoco el sistema de gestión administrativa que hará posible su ejecución. Si el modelo de administración municipal y el desempeño de algunos funcionarios y funcionarias no permiten satisfacer las necesidades de la ciudadanía, habrá que ser irruptores para generar cambio organizacional alineado con la política. En este sentido, se reconoce que las organizaciones públicas y privadas están sometidas constantemente a procesos de mejoramiento por razones históricas o coyunturales y están influenciadas por factores internos y externos.

Es necesario que el Gobierno local reflexione sobre su actuar y defina una visión clara con respecto al uso y aprovechamiento del espacio público, para lo cual puede realizar un diagnóstico organizacional, definir los cursos de acción a partir de una política, fortalecer los valores de la municipalidad, definir actividades, establecer cronogramas, optimizar la estructura y sensibilizar al personal sobre la importancia que tienen los espacios públicos para promover mayor desarrollo social, económico, cultural y político en el cantón. Todas estas acciones contribuirán al desarrollo de la política pública, que sería ejecutada por la caja negra de la administración, en la que están presentes el clima organizacional, el capital humano, los procesos, el estilo de la Dirección y el presupuesto. 
Por lo anterior, se considera conveniente valorar la creación de una Oficina de Proyectos para el Desarrollo Comunal, integrada por un equipo interdisciplinario conformado por el personal que actualmente trabaja en la Dirección de Planificación Urbana, Catastro y la Oficina de Desarrollo Social, con el fin de acercar el Gobierno local a las comunidades y lograr mayor proactividad, acompañamiento e integración durante el diseño de los proyectos, mejorar la coordinación interna $y$ analizar los proyectos con un enfoque social sustentado en criterios de pertinencia, efectividad, economía, cobertura y sostenibilidad en función de las demandas las comunidades. Las autoridades municipales hoy tienen que adoptar posiciones proactivas y superar la fase, característica del siglo pasado, de ser ejecutores de obras. Los distintos cuerpos municipales deben asumir el rol que les corresponde en materia de gobernabilidad y gobernanza. Solo en una estrecha interacción con las comunidades, con la ciudadanía se logra fortalecer la legitimidad del ejercicio del poder político.

Con el fin de determinar la pertinencia de los proyectos que se consideran necesarios para recuperar los espacios públicos cantonales, es fundamental identificar los problemas de manera adecuada y valorar las distintas alternativas de solución. Uno de los factores esenciales para actuar de forma acertada frente a un problema es la escogencia de la mejor opción que permita solucionarlo. El punto de partida es una correcta valoración en la fase de problematización de la situación, previa a la formulación de una política pública. Para ello es necesario apoyarse en una metodología adecuada y recopilar suficiente información que permita entender la situación que requiere atender. En este sentido, el árbol de problemas es un proceso analítico que permite identificar la problemática central, así como mapear las causas y los efectos para justificar la intervención. Ello como un instrumento a utilizar en el marco del ciclo de políticas públicas (formulación, implementación y evaluación).

A partir de lo anterior, es necesario definir y comparar las diferentes opciones de solución, así como la selección de la alternativa que se va a desarrollar, definiendo los objetivos y resultados esperados, al igual la vinculación con las políticas, planes y estrategias de desarrollo a nivel nacional, sectorial, regional y local para lograr desarrollo social, económico, ambiental e institucionalterritorial. No hay que perder de vista que tanto el distrito como el cantón forman parte de un complejo entramado que da lugar al Estado; y este interactúa con otros actores estatales y no estatales internacionales que inciden en la dinámica comunitaria.

Por otra parte, para mejorar la gestión del Gobierno local y tener éxito en las decisiones asociadas con la política de espacios públicos, se requiere considerar los factores formales de la organización (procedimientos, manuales, lineamientos, la estructura organizacional y los recursos disponibles) y los informales (costumbres, hábitos, experiencia). Además, es necesario conocer y administrar los factores externos de la organización, tales como: las necesidades de los usuarios, la relación con los proveedores de bienes y servicios, la situación económica del país y las amenazas que puedan afectar el adecuado aprovechamiento de los espacios públicos.

Para ajustar la gestión institucional de la Municipalidad de Montes de Oca que permita la recuperación de espacios públicos, se requiere fijar una meta de mediano y largo plazo por medio de la formulación e implementación de una política, optimizar los procesos y procedimientos que permitan ejecutarla de manera coordinada y contar con los recursos materiales, humanos $y$ presupuestarios necesarios para brindar un mejor servicio. Se requiere propiciar una vocación de servicio público fomentando la meritocracia y teniendo presente que las y los funcionarios públicos deben orientar el accionar hacia la generación de valor público.

La decisión de las autoridades político-administrativas de formular e implementar una política cantonal de recuperación de espacios públicos y la participación de actores sociales del cantón, son fundamentales para iniciar un proceso de diálogo, discusión y de construcción de acuerdos que permitan avanzar hacia la consolidación de una identidad cantonal.

Previo a la formulación e implementación de una política cantonal de recuperación de espacios públicos, es necesario definir una agenda que puede ser construida a partir del modelo 
pluralístico, el cual reconoce la importancia de la participación de diferentes grupos con el objetivo de plasmar las necesidades y aspiraciones de los ciudadanos, del sector empresarial del cantón, de los dirigentes comunales $y$ las autoridades municipales, potenciando las capacidades administrativas $y$ organizativas del Gobierno local. De esta manera, la agenda sentaría las bases para la formulación e implementación de la política cantonal de espacios públicos.

Por último, se reconoce el esfuerzo que realiza la Comisión de Espacios Públicos de la Municipalidad de Montes de Oca por promover la participación de la comunidad y construir una política pública de espacios públicos que responda a las necesidades del cantón; no obstante, no debe obviarse que la toma de decisiones recae en las autoridades municipales quienes definen las prioridades cantonales y la asignación de recursos.

\section{CONSIDERACIONES FINALES}

La compleja dinámica social, política, económica, cultural y jurídica de inicios del siglo XXI, junto con el acelerado proceso de urbanización y desarrollo de metrópolis, pensadas en función de una galopante actividad comercial centrada en espacios no públicos, como los centros comerciales o "malls", ha dado lugar a retos y desafíos sobre la identidad comunitaria y la participación ciudadana. Ello ha transformado el espacio público, alejándose cada vez de ser el lugar de encuentro para construir cohesión social; siendo sustituido por escenarios despersonalizados, en los que las personas no se comunican entre sí y menos debaten sobre los problemas colectivos, sino que están absortas en las redes sociales, viviendo lo que se conoce como las "proximidades distantes".

Por ello, Venturini (2004) alude al desarrollo de centros urbanos y su relación con el patrimonio e identidad de ciudadana en el mundo globalizado de esta nueva centuria. Pero ello significa una ruptura con el espacio público de las ciudades tradicionales, que el que "...da identidad y carácter a la ciudad, el que permite reconocerla y vivirla en sus sitios urbanos: naturales, culturales y patrimoniales", dando lugar a partir de la década de 1990 a la idea de "espacio privado de uso público" (Perahia, 2007).

Esto tiene que ver con la naturaleza y concepción del territorio, que no puede seguir siendo concebido únicamente en la dimensión nacional y de la relación entre espacio público-vida privada (de Piero, 2003). En el primer aspecto hay que entender que la dimensión territorial nacionalestatal tiene, por un parte, aquella que corresponde a lo local-cantonal $y$, por otra, a la regionalinternacional. Eso genera nuevos modos de hacer política (de Piero, 2003), de construir identidad y ciudadanía -sobre todo en términos de participación-.

De esa forma se desvirtúa el espacio público como punto de encuentro para construir identidad y fomentar la participación ciudadana. Esto se hace más evidente en comunidades con poblaciones que han inmigrado en función de otro tipo de prioridades, como es el estudio o el trabajo. Esto porque ese ciudadano o ciudadano convierte su comunidad de dormitorio, sin integrarse como miembro de la comunidad. Esto agrega más carga a las autoridades municipales, que en el caso de Costa Rica históricamente se han considerado ejecutores de obras y no gestión de lo público, en un sentido amplio.

\section{REFERENCIAS}

Ávila, M. (2017). Construcción de identidad comunitaria por medio de la Estrategia Socio Deportiva. [Tesis de licenciatura, Universidad Alberto Hurtado]. https://repositorio.uahurtado.cl/ handle/11242/23816

Borja, J. (2001). La ciudad y la nueva ciudadanía. Barcelona: Organización de Estados 
Iberoamericanos. Recuperado el 31 de agosto de 2019, de https:/www.oei.es/historico/ cultura/LaciudadJBorja2.htm

Borja, J. \& Muxí, Z. (2003). Espacio público: ciudad y ciudadanía. Editorial Electa.

Chávez, D. (2013). El parque del barrio. La Ciudad Paralela. https://web.archive.org/ web/20200217045632/http://www.laciudadparalela.com/el-parque-del-barrio/

Cunha, J. (2002). Urbanización, redistribución espacial de la población y transformaciones socioeconómicas en América Latina. CEPAL. https://repositorio.cepal.org/handle/11362/7168

Dammert, L. (2004) ¿Ciudad sin ciudadanos? Fragmentación, segregación y temor en Santiago. Revista EURE - Revista de Estudios Urbano Regionales, 30(91), 87-96. http://www.eure.cl/ index.php/eure/article/view/1282

de Piero, S. (2003). Pensando el espacio público en la globalización: cuatro reflexiones. Polis. Revista Latinoamericana, 4. https://journals.openedition.org/polis/7055

Di Siena, D. (2010). Redes sociales, identidad digital y espacio público. Blog La Ciudad Viva. Recuperado el 2 de setiembre de 2019, de https://ecosistemaurbano.org/castellano/redessociales-identidad-digital-y-espacio-publico/

Duhau, E \& Giglia, Á (2010). El espacio público en la Ciudad de México. De las teorías a las prácticas. En Los grandes problemas de México. Desarrollo urbano y regional. T-II (pp. 389 448). Colegio de México.

Estévez, A. (2016). ¿Derechos humanos o ciudadanía universal? Aproximación al debate de derechos en la migración. Revista Mexicana de Sociología, 78(1). 61-87.

Fumero, P. (2009). La ciudad fragmentada: la Gran Área Metropolitana (GAM). Revista Herencia, 22(2), 7-12. http://revistas.ucr.ac.cr/index.php/herencia/article/view/3532

Fundación Promotora de Vivienda. (2003). Construyendo Ciudadanía Social e Identidad Barrial. Recuperado el 31 de agosto de 2019, de https://www.fuprovi.org/wp-content/uploads/2018/02/ Proy_Ciudad_e_Identidad_Barrial_I_Fase_Los_suenos_no_pesan_pero_si_cuestan.pdf

Fuprovi. (2018). Informe nacional: Situación de la vivienda y desarrollo urbano en Costa Rica 2017. Recuperado el 5 de setiembre de 2019, de https://www.fuprovi.org/wp-content/ uploads/2018/02/Situacio\%CC\%81n-de-la-Vivienda-y-Desarrollo-Urbano-en-CostaRica-2018-1.pdf

Gallegos, M. (2011). La ciudad en declive: expresión de la crisis de lo urbano. Herramienta. Revista de debate y crítica marxista, No. 48. Recuperado el 31 de agosto de 2019, de https:// herramienta.com.ar/articulo.php?id=1584

Magalhães, F. (2014, marzo 11). ¿Necesitamos espacios públicos en la era de las redes sociales? Ciudades Sostenibles. Recuperado el 12 de julio de 2019, de https://blogs.iadb.org/ciudadessostenibles/es/necesitamos-espacios-publicos-en-la-era-de-las-redes-sociales/

Mazza, A. (2009). Ciudad y espacio público. Las formas de la inseguridad urbana. Cuadernos de Investigación Urbanística, 62. http://polired.upm.es/index.php/ciur/article/view/278

Ministerio de Planificación Nacional y Política Económica (2017). Costa Rica: Índice de Desarrollo Social (IDS) 2017. Recuperado el 13 de julio de 2019, de https://documentos.mideplan.go.cr/ share/s/L0GWBZnfRceDJjpxwm7zFQ

Perahia, R. (2007). Las ciudades y su espacio público. Ponencia presentada al IX Coloquio Internacional de Geocrítica "Los problemas del mundo actual, soluciones y alternativa desde la geografía y las ciencias sociales", Porto Alegre, 28 de mayo a 1 de junio. Recuperado el 12 de julio de 2019, de: http://www.ub.edu/geocrit/9porto/perahia.htm

Pindado, F. \& Rebollo, O. (2015). El debate público. Barcelona: Escola de l'IGOP. Recuperado el 11 de julio de 2019, de https://igop.uab.cat/wp-content/uploads/2015/04/DEBATE_PUBLICO_2015_ A4-IGOP.pdf 
Prochile Costa Rica. (2018). Tendencias del Mercado. Construcciones verticales en el mercado de Costa Rica. Recuperado el 2 de setiembre de 2019, de https://www.prochile.gob.cl/wp-content/ uploads/2018/07/tendencias_construcciones_verticales_costa_rica_2018.pdf

Programa de las Naciones Unidas para el Desarrollo. (2018). Objetivos de Desarrollo Sostenible. Recuperado el 12 de agosto de 2018, de http://www.undp.org/content/undp/es/home/ sustainable-development-goals/goal-11-sustainable-cities-and-communities.html\#targets

Programa Estado de la Nación en Desarrollo Humano Sostenible. (2013). Indicadores Cantonales Censos Nacionales de Población y Vivienda 2000 y 2011. http://repositorio.conare.ac.cr/ handle/20.500.12337/882

Rosas-Baños, M. (2013). Nueva Ruralidad desde dos visiones de progreso rural y sustentabilidad: Economía Ambiental y Economía Ecológica. Polis. Revista Latinoamericana, 34. http:// journals.openedition.org/polis/8846

Sánchez, D. \& Domínguez, L. (2014). Identidad y espacio público: ampliando ámbitos y prácticas. Editorial Gedisa.

Segovia, O (2007). Espacios públicos urbanos y construcción social: una relación de correspondencia. En O. Segovia (Ed.), Espacios públicos y construcción social. Hacia un ejercicio de ciudadanía (pp. 15-28). Ediciones Sur.

Vargas, D. \& Merino, M. (2012). El papel de los espacios públicos y sus efectos en la cohesión social: experiencia de la política pública en México. Estudios sociológicos, 30(90): 897-914.

Venturini, E. J. (2004). Centros urbanos, patrimonio e identidad ciudadana en el mundo de la globalización. Estudios digital. 15, 157-168. https://doi.org/10.31050/1852.1568.n15.13543

Vicherat, D. (2007). ¿Qué tienen en común la identidad, el espacio público y la democracia? Algunas reflexiones para los conceptos. Instituto Universitario Europeo.

Zúñiga, R., Corrales, O., \& Vargas, M. (2018). Reporte de situación Costa Rica 2018. $2^{a}$ edición. Poder Judicial. 